\title{
Answers to quiz - Respiratory medicine
}

DOI: http://doi.org/10.4038/jccp.v47i2.7796

Q1.

a) Pulmonary embolism.

b) The A-a gradient is increased in keeping with a ventilation perfusion mismatch.

c) Bilateral venous compression ultrasound (CUS) may help identify a deep venous thromboembolism allowing sufficient evidence to start treatment.

d) V/Q scan or CT-pulmonary angiography (CTPA).

It is important to consider radiation exposure to mother and child.

CTPA increases the maternal background $0.1 \%$ risk of breast cancer within the next $10 \mathrm{yrs}$ by $13.6 \%$. Due to radiation exposure in utero V/Q scan carries a higher risk of childhood cancer of 1/280,000 compared to CTPA of less than $1 / 1000,000$. However overall exposure and risk with V/Q scan is minimal.

e) Low molecular weight heparin to be continued for 6 weeks postnatally and for a minimum of 3 months in total.

Q2.

a) Restrictive lung disease. The reduced TLco suggests a parenchymal abnormality.

b) Sub-pleural honeycombing and evidence of traction bronchiectasis in keeping with pattern of usual interstitial pneumonia (UIP).

c) Diagnosis: Idiopathic pulmonary fibrosis (IPF).

Differential: Asbestosis, rheumatoid-interstitial lung disease. Both are less likely as there is no history of asbestos exposure or symptoms/signs supportive of rheumatoid disease.

d) Anti-fibrotic agents pirfenidone which is an immunosuppressant and nintedanib which is a tyrosine kinase inhibitor have been licensed by NICE for the treatment of IPF in patients with an FVC between $50-80 \%$. It is recommended that treatment be stopped if FVC falls by $10 \%$ over 12 months.

Q3.

a) Patchy bilateral opacities centred around the associated bronchus (i.e. 'bronchocentric').

b) Cryptogenic oraganising pneumonia (COP) is the most likely diagnosis.

Bacterial infection is unlikely in view of the prolonged history and no response to antibiotics. Bronchoalveolar carcinoma, pulmonary lymphoma are among the differentials but the clinical presentation is not consistent. Eosinophilic pneumonia is unlikely as eosinophil count is normal.

c) Bronchoscopy with transbronchial biopsies. This will allow exclusion of other causes including malignancy.

Typical histopathological pattern of COP is characterised by proliferation of granulation tissue within the small airways known as intra-alveolar buds.

If transbronchial biopsy is unsuccessful VATS biopsy should be considered.

d) COP generally responds well to corticosteroids. A reasonable regime is as follows:

$0.75 \mathrm{mg} / \mathrm{kg}-1$ prednisone daily during 4 weeks, followed by $0.5 \mathrm{mg} / \mathrm{kg}-1$ for 4 weeks, then $20 \mathrm{mg}$ for 4 weeks, $10 \mathrm{mg}$ for 6 weeks, and then $5 \mathrm{mg}$ for 6 weeks before stopping. Optimal duration of therapy is unknown but is usually required for at least 3-12 months. 